\title{
Influence of Grain Size on the Band-gap of Annealed SnS Thin Films
}

\author{
Priyal Jain ${ }^{a, b}$ and P. Arun ${ }^{b *}$ \\ ${ }^{a}$ Department of Electronic Science, \\ University of Delhi, South Campus \\ Delhi 110021 \\ ${ }^{b}$ Material Science Research Lab, \\ S.G.T.B. Khalsa College, \\ University of Delhi, Delhi - 110 007, India
}

November 7, 2012

\begin{abstract}
The manuscript reports the variation in optical band-gap of vacuum annealed SnS thin films. The samples were characterized by using X-Ray Diffraction, Scanning Electron Microscopy (SEM), UV-visible Spectroscopy and Raman Analysis. Results show that while annealing does not effect the nano-crystalline sample's lattice structure or unit cell size, it does control the grain size. The band-gap $\left(\mathrm{E}_{\mathrm{g}}\right)$ decreases with increase in grain size. $\mathrm{E}_{\mathrm{g}}$ values were found to be very high (1.8-2.1 eV) for samples studied.
\end{abstract}

*email:arunp92@physics.du.ac.in, Telephone:091 011 29258401, Fax: 09101127666220 


\section{Introduction}

Tin sulphide ( $\mathrm{SnS})$ is a semiconducting material formed from elements belonging to the IV-VI group of the periodic table. Research on $\mathrm{SnS}$ shows that it has potential use as holographic or optical data storage medium 1, 2]. Also, due to its high absorption coefficient (of the order $\sim 10^{4} \mathrm{~cm}^{-1}$ ) and bandgap $\sim 1.3-1.6 \mathrm{eV}$, SnS films have been used as photovoltaic devices [3, 4. SnS exists in various crystallized states like orthorhombic [5], tetrahedral (Zinc blende like) [6] or a highly distorted rock-salt $(\mathrm{NaCl})$ structure [7. Due to the nature of the tin and sulphur bondings, it forms two dimensional sheets [8], giving rise to a layered structure with strong intra-planar forces and weak Van der Waal forces between the adjacent planes [9, 10. Films of SnS have been fabricated using various techniques such as thermal evaporation [11, RF sputtering [12, chemical vapour deposition [13], electrodeposition [14, spray pyrolysis [15] and sulphurisation of metallic precursors [16]. The optical properties of SnS strongly depend on the deposition method used [17]-[21]. While a maximum band gap $(1.78 \mathrm{eV})$ was reported in films obtained by wet chemical method [5], the lowest band-gap $(1.12 \mathrm{eV})$ was reported for films made by chemical bath deposition [17].

The deposition techniques influences the film's grain size, crystal structure and residual strain which in turn effect the optical properties of the film. In light of the increasing importance of SnS and its potential applications, it becomes important to study the film structure with its band-gap. Thus, in this manuscript we report our studies on vacuum evaporated SnS thin films that were vacuum annealed after fabrication and try to establish a relation between their structural and optical properties.

\section{Experimental}

SnS films of varying thicknesses were fabricated by thermally evaporating powdered SnS at vacuum better than $\sim 4 \times 10^{-5}$ Torr in a Hind High Vac (12A4D) thermal evaporation coating unit. The depositions were made on glass microscopy slides maintained at room temperature. SnS powder of $99 \%$ purity supplied by Himedia(Mumbai) was used as the starting material. The thickness of the asgrown films were measured by a Veeco Dektak Surface Profiler (150). The samples were then subjected to post-annealing temperatures under vacuum for 30 mins at $373 \mathrm{~K}$ and $473 \mathrm{~K}$. The structural, morphological, compositional and optical characterisations were done systematically for both the as grown and vacuum annealed films. We report and compare here the structural, morphological, compositional and optical properties of annealed samples 
of five different thicknesses, 270, 480,600, 650 and $900 \mathrm{~nm}$. The stuctural analysis were done using Bruker D8 Diffractometer. The Raman spectra of the films were obtained with Renishaw Invia Raman Microscope using Argon laser beam in the reflection mode. The surface morphology of the samples and their chemical composition were examined with FEG-SEM JSM7600F and its Energy-dispersive X-Ray Spectroscopy (EDX) attachment. The optical properties of the samples were carried out using Systronics UV-VIS Double beam Spectrophotometer (2202).

\section{Results and Discussion}

The Raman spectra were collected from back scattered $\mathrm{Ar}^{2+}$ laser. Analysis of all our samples were done maintaining constant beam area, exposure time $(30 \mathrm{sec})$ and power $(15 \mathrm{~mW})$. All Raman spectra were collected using low power laser since studies on $\mathrm{SnS}$ as an optical data storage medium have shown changes induced in it by photo-thermal absorption of light. All the samples without exception showed three peaks, two prominent peaks around $170 \mathrm{~cm}^{-1}$ and $238 \mathrm{~cm}^{-1}$ and a third peak of lower intensity at $330 \mathrm{~cm}^{-1}$. The $330 \mathrm{~cm}^{-1}$ peak is associated with $\mathrm{SnS}_{2}\left[22\right.$. The $\sim 170$ and $238 \mathrm{~cm}^{-1}$ Raman peaks are associated with those of single crystal $\mathrm{SnS}[\mathbf{5}$. Our peak positions are slightly displaced from these positions. Figure (1) shows representative Raman spectra of a 900nm thick film, before annealing (rt) and after annealing at $373 \mathrm{~K}$ and $473 \mathrm{~K}$ respectively. The relative contribution of the $330 \mathrm{~cm}^{-1}$ peak in annealed samples is lower as compared to that in as grown samples. Annealing, hence, removes $\mathrm{SnS}_{2}$ bonding/ defects formed during film fabrication. The $170 \mathrm{~cm}^{-1}$ or

the $\mathrm{B}_{2 \mathrm{~g}}$ mode's peak corresponds to interaction along the inter-layer ' $\mathrm{b}$ ' axis, while the $238 \mathrm{~cm}^{-1}$ peak is the $\mathrm{A}_{\mathrm{g}}$ mode that corresponds to the symmetric Sn-S bonding stretching mode in the a-c plane [23. It is clear from figure (1) that the relative contributions from $\mathrm{B}_{2 \mathrm{~g}}$ and $\mathrm{A}_{\mathrm{g}}$ do not change on annealing.

Figure (2) exhibits the X-Ray diffractograms of thin annealed (473 K) SnS films of different thicknesses. Three prominent peaks are seen at $2 \theta \sim 31^{\circ}, 37^{\circ}$ and $43^{\circ}$. These peak positions matched with those reported in ASTM Card No. 83-1758. The SnS in our samples hence exists with orthorhombic unit cell structure with the (040) peak having the maximum intensity. While there are many works in the literature that show (040) peak to be the most intense [24, 25], others have reported (111) peak to be the most intense [5, 26]. This again underlines how growth technique and conditions influence the character of the SnS films. The Miller indices of the remaining prominent peaks are also indicated in the figure. The lattice parameters 'a', 'b' and 'c' of $\mathrm{SnS}$ in single crystal state are given as $4.148,11.48$ and $4.177 \AA$, respectively. 
We have evaluated the lattice parameters from the (040), (200) and (131) peak's position using the relation

$$
\sin ^{2} \theta_{i}=A h_{i}^{2}+B k_{i}^{2}+C l_{i}^{2}
$$

The lattice parameters of the annealed samples were found to be $4.07 \pm 0.002,11.36 \pm 0.01$ and $4.45 \pm 0.002 \AA$. They were found to be different from those of SnS in single crystal state. While the variation in 'a' and 'b' maybe be considered marginal (decrease), there is a significant increase in 'c'. Albanesi et al [10] have done systematic analyses to understand the effect of changing unit cell size on SnS band-gap. Their study suggests that an increase in volume of the unit cell would lead to a lowering in SnS band-gap. We shall return to this when we discuss the results of our optical studies. While there is a significant change in the lattice constants (namely 'c') as compared to that of single crystal, we find that they do not change with film thickness or annealing temperature. Figure (3A) shows all three lattice parameters remaining uneffected with film thickness. Since points coincide, each point in this graph represents samples annealed at $373 \mathrm{~K}$ and $473 \mathrm{~K}$. The difference in lattice constant present $\left(\mathrm{c}_{\mathrm{obs}}\right)$ with respect to that given in the ASTM $\left(\mathrm{c}_{\mathrm{ASTM}}\right)$ implies that the grains are in a state of stress with the peaks shifting left compared to those reported in the ASTM, we conclude tensile residual stresses are acting within the grains [27. However, $\mathrm{c}_{\mathrm{obs}}$ is constant for all the samples hence the same tensile residual stress exists in all the samples. This tensile residual stress thus appears as a background in our study. While the lattice parameters remain unaltered, the grain size changes with annealing. The grain size reported were calculated using the Full Width at Half Maxima (FWHM) of the diffraction peaks in Scherrer's formula 28. From figure (3B), it is clear that there is a presistent increase in grain size with annealing, however this effect is more pronounced in thinner films. The grain size increases from $12 \mathrm{~nm}$ to $25 \mathrm{~nm}$ in the $270 \mathrm{~nm}$ films, while this increase in grain size with annealing temperature is impeded for thicker films (fig 3B).

$\mathrm{SnS}$ is a layered compound with zig-zagged molecules of $\mathrm{SnS}$ forming layers. These layers stack one on top of the other along the 'b' axis with weak Van der Waal 'inter-planar' forces acting between the layers. The layers themselves because of the zig-zagging have finite thickness and along the 'b' axis have strong 'intra-planar' forces acting within them. Ehm [29] has shown that the application of pressure decreases inter-planar distances along 'b' axis without disturbing intra-planar spacing. As stated above, the Raman $\mathrm{B}_{2 \mathrm{~g}}$ peak represents vibrations along the 'b' axis. Fig (4) shows the variation of Raman peak position of annealed samples. While the $A_{g}$ peak remains fixed at $226.5 \mathrm{~cm}^{-1}$, the $\mathrm{B}_{2 \mathrm{~g}}$ peak shows a systematic shift to a higher wavenumber with increasing 
film thickness. It would appear thinner films with larger grain size have vibrations taking place at lower wavenumber than thicker films with smaller grains. The Raman peak's position and its FWHM is a rich source of information. While it does give information on the structure, the corroborative X-ray diffraction study showing no variation in lattice parameters imply variation in Raman spectra is indicative of something else. Literature on Raman Analysis have shown variation of FWHM to be a measure of phonon confinement [30, 32] with shift in peak position related to grain size [32]-[36] and nature of defect on the grain boundaries [30]. A shift to higher wavenumbers is indicative of smaller grain size. Hence, the results of X-Ray diffraction and Raman Analysis, when put together, suggest that annealed $\mathrm{SnS}$ thin films have large grains with grain size decreasing as the annealed film thickness increases. This is best understood from fig (5).

The results shown in figure (3B) are also reflected in the Scanning Electron Microscope's micrographs (fig 6). The film of $480 \mathrm{~nm}$ thickness showed significant increase in grain size and grain density when annealed at higher temperature as compared to the $900 \mathrm{~nm}$ thick film. Comparing the $900 \mathrm{~nm}$ thick films annealed at $373 \mathrm{~K}$ and $473 \mathrm{~K}$ respectively, we do not find such improvement in grain size or density as was infered from the X-Ray diffraction analysis. Fig (7) shows EDX of $900 \mathrm{~nm}$ thick film annealed at $473 \mathrm{~K}$. Even after annealing at $473 \mathrm{~K}$ no oxidation is evident ( $\mathrm{Si}$ and $\mathrm{O}$ is from glass substrate). This is evidently due to fact annealing was done in vacuum $\left(5 \times 10^{-2}\right.$ Torr $)$. Based on the structural and morphological results, it would be interesting to study the optical properties of these samples. Since the lattice parameters and residual tensile stress on the grain's bulk remains same with only grain size and to an extent grain density varying in the samples, the study should reveal the contribution that grain size have on the properties like band gap, $\mathrm{E}_{\mathrm{g}}$.

The optical absorption and transmittance spectra of all the samples were obtained in between wavelength range 300-900 nm. The absorption spectras were typical with absorption increasing near band-edge. Tin sulphide is known to have both direct band-gap [37] and indirect band-gap [5]. The band-gap can be evaluated using the absorption coefficient $(\alpha)$ obtained from the UV-visible spectra. The band-gap is obtained using the relation 38

$$
\alpha h \nu=\left(h \nu-E_{g}\right)^{n}
$$

where ' $n=1 / 2$ ' for direct allowed transitions. On extending the best fit line on the linear part of the curve between $(\alpha \mathrm{h} \nu)^{2}$ and $\mathrm{h} \nu$, the point where the line cuts the 'X-axis' gives the energy band-gap of the sample. Using this method, we have evaluated band-gaps for all the samples. As discussed earlier, the lattice parameters 
remained unchanged for annealed films, hence it can be conclusively said that the bandgap variation is not caused by the lattice defects. The bandgap of SnS films strongly depends on the depostition process and the film parameters like the film thickness. SnS amorphous film of thickness $1000 \mathrm{~nm}$ grown on a glass substrate by spray pyrolysis has a bandgap of $1 \mathrm{eV}$ [15]. While films grown by vacuum evaporation were found to be nanocrystalline with bandgap varying between 1.15-1.3 eV. The variation in bandgap was found to be related to the distance between the source and the substrate [26]. Films grown on ITO substrates by pulsed electrodeposition were polycrsytalline in nature with bandgap being $1.34 \mathrm{eV}$ [24]. Polycrystalline films grown by chemical bath deposition on glass substrates of $290 \mathrm{~nm}$ thickness also possess low bandgap $(1.15 \mathrm{eV})$ [17. While the films grown with RF sputtering technique are also nano-crystalline, they have very high bandgaps varying from 1.3 to $1.7 \mathrm{eV}$ [12]. A recent study [39] on $\mathrm{SnS}$ polycrystalline thin films grown by thermal evaporation reports $\mathrm{SnS}$ band-gap between 2.15-2.28 eV. Clearly, polycrystalline $\mathrm{SnS}$ films have higher band-gaps compared to their amorphous counterparts.

Figure (8) shows the grain size dependence of the bandgap. The bandgap decreases with the increasing grain size. Table 1 summarizes the findings of this study. It shows on annealing, thinner films have larger grains. The large grain size gives the annealed samples a lower band-gap. Thicker films however, have smaller grains and an enhanced band-gap. The bandgap of a sample is broadly effected by its chemical composition, crystal structure, grain size and defects. Of all these contributing parameters, except for grain size, all other parameters remain constant. While the larger volume of the unit cell leads to a background low $\mathrm{E}_{\mathrm{g}}(\sim 1.8 \mathrm{eV})$ as explained by Albanesi [10, decreasing grain size contribute to an increase in $\mathrm{E}_{\mathrm{g}}$ of the vacuum annealed SnS samples. The band-gap's variation with grain size due to quantum confinement has the quantitative form [40]

$$
E_{g}^{n a n o}=E_{g}^{b u l k}+\frac{\hbar^{2} \pi^{2}}{2 M r^{2}}
$$

where ' $r$ ' is the radius of the nanoparticle and 'M' the effective mass of the system. The solid line of fig (8) shows good fit of eqn(1) to our experimental data points. The result again reiterates influence of both the lattice parameters (via $\mathrm{E}_{\mathrm{g}}^{\mathrm{bulk}}$ ) and grain size on the band-gap of $\mathrm{SnS}$. 


\section{Conclusion}

Thin films of SnS were fabricated by thermal evaporation on microscopy glass slides at room temperature. These films when annealed in vacuum at $373 \mathrm{~K}$ and $473 \mathrm{~K}$ developed a tensile strain along the lattice 'ac' plane. The magnitude of this tensile strain was independent of the film thickness and annealing temperature. However, the film thickness and annealing temperature play an important influence on the samples grain size. We conclude from our results that $\mathrm{E}_{\mathrm{g}}$ of nano-crystalline $\mathrm{SnS}$ films invariably depends on the grain size due to quantum confinement.

\section{Acknowledgment}

Authors are thankful to the Department of Science and Technology for funding this work under research project SR/NM/NS-28/2010. We are also grateful to Dr Chhaya Ravi Kant, Department of Applied Sciences, IGIT (GGS-IP University, Delhi) for extending necessary assistance.

\section{References}

[1] M. Radot, Rev. Phys. Appl., 18, (1977) 345 .

[2] S.G. Patil and R.H. Tredgold, J. Pure Appl. Phys., 4, (1971) 718.

[3] Zhijie Wang, Shengchun Qu, Xiangbo Zeng, Junpeng Liu, Changsh Zhang, Furui Tan Lan Jin and Zhanguo Wang, Journal of Alloys and Compounds, 482 (2009) 203.

[4] H. Noguchi, A. Setiyadi, H. Tanamora, T. Nagatomo and O. Omato, Sol. Energy Mater. Sol. Cells, 35 (1994) 325 .

[5] S. Sohila, M. Rajalakshmi, Chanchal Ghosh, A.K. Arora and C. Muthamizhchelvan, Journal of Alloys and Compounds, 509 (2011) 5843.

[6] C. Gao, H. Shen, T. Wu, L. Zhang and F. Jiang, Mater. Lett., 64 (2010) 2177.

[7] A.N. Mariano and K.L. Chopra, Appl. Phys. Lett., 10 (1967) 282

[8] T. Jiang and G A Ozin, J. Mater. Chem., 8 (1998) 1099. 
[9] P.M. Nikolic, P. Lj Miljkovic, B. Mihajlovic and B. Lavrencic, J. Phys. C: Solid Status Phys., 10 (1977) L289.

[10] L. Makinistian and E.A. Albanesi, Comput. Mater. Sci., 50 (2011) 2872.

[11] M.M.E.I. Nahass, N.M. Zeyada, M.S. Aziz and N.A.E.I. Ghamaz, Opt. Mater., 20 (2002) 159.

[12] Katy Hartman, J.L. Johnson, Mariana I. Bertoni,Daniel Recht, Micheal J. Aziz, Micheal A. Scarpulla and Tonio Buonassis, Thin Solid Films, 519 (2011) 7421.

[13] M.T.S. Nair and P.K. Nair, Semicond. Sci. Technol., 6 (1991) 132.

[14] A. Ghazali, Z. Zainal, M.Z. Hussein and A. Kassim, Sol. Energy Mater. Sol. Cells, 55 (1998) 237.

[15] B. Thangaraju and P. Kaliannan, J. Phys. D: Appl. Phys., 33 (2000) 1054.

[16] K.T. Ramakrishna Reddy and P. Purandhara Reddy, Mater. Lett., 56 (2002) 108.

[17] Chao Gao, Honglie Shen and Lei Sun, Appl. Surf. Sci., 257 (2011) 6750.

[18] K.T. Ramakrishna Reddy, P. Purandhara Reddy, P.K. Datta and R.W. Miles, Opt. Mater., 17 (2001) 295.

[19] G.H. Yue, D.L. Peng, P.X. Yan, L.S. Wang, W. Wang and X.H. Luo, Journal of Alloys and Compounds, 468 (2009) 254.

[20] M Devika, N Koteeswara Reddy, K Ramesh, K R Gunasekhar, E S R Gopal and K T Ramakrishna Reddy, Semicond. Sci. Technol., 21 (2006) 1125.

[21] A. Ortiz, J.C. Alonso, M. Garcia and J. Toriz, Semicond. Sci. Technol., 11 (1996) 243.

[22] G. Lucovsky, J. C. Mikkelsen, W. Y. Liang, R. M. White and R. M. Martin, Phys. Rev. B, 14 (1976) 1664 .

[23] H.R. Chandrasekhar, R.G. Humphreys, U. Zwick and M. Cardona, Phys. Rev. B, 15, (1977) 2177.

[24] G.H. Yue, W. Wang and L.S. Wang, Journal of Alloys and Compounds, 474 (2009) 445.

[25] Feng Jiang, Honglie Shen, Chao Gao, Bing Liu, Long Lin and Zhou Shen, Appl. Surf. Sci., 257 (2011) 4901. 
[26] M. Devika, N. Koteeswara Reddy, D. Sreekantha Reddy, S. Venkatramana Reddy, K Ramesh, E S R Gopal, K R Gunasekhar, V Ganesan and Y B Hahn, J. Phys.: Condens. Mater., 19 (2007) 1.

[27] A.L. Patterson, Phys. Rev., 56 (1956) 978.

[28] B.D. Cullity and S.R. Stock, "Elements of X-Ray Diffraction", $3^{\text {rd }}$ Ed., Prentice-Hall Inc (NJ, 2001).

[29] L. Ehm, K. Knorr, P. Dera, A. Krimmel, P. Bouvier and M. Mezouar, J. Phys.:Condens. Mater., 16 (2004) 3545 .

[30] Kuninori Kitahara, Toshitomo Ishii, Junki Suzuki, Takuro Bessyo and Naoki Watanabe, INT J. Spectrosc., 2011 (2011) 1.

[31] Zhu J.S., Lu X.M., Jiang W., Tian W., Zhu M., Zhang M.S., Chen X.B., Liu X. and Wang Y.N., J. Appl. Phys., 81 (1997) 1392.

[32] Hyun Chul Choi, Young Mee Jung and Seung Bin Kim, J. Of Vib. Spectrosc., 37 (2005) 33.

[33] Rajalakshmi M, Arora A.K., Bendre B.S. and Mahamuni S, J. Appl. Phys., (2000) 872445.

[34] Yang C.L., Wang J.N., Ge W.K., Guo L., Yang S.H. and Shen D.Z., J. Appl. Phys., (2001) 904489.

[35] Guo L, Yang S, Yang C, Yu P, Wang J, Ge W and Wong G.K.L., Appl. Phys. Lett., (2000) 762901.

[36] Alim K.A., Fonoberrov V.A. and Baladdin A.A., Appl. Phys. Lett., (2005) 86053103.

[37] M. Devika, N. Koteeswara Reddy, M. Prashantha, K. Ramesh, S. Venkatramana Reddy, Y.B. Hahn and K.R. Gunasekhar, Phys. Status Solidi A, 207, No. 8, (2010) 1864.

[38] K. Kamano, R. Nakata and M. Sumita J Phys. D: Applied Physics, 22 (1989) 136.

[39] Shuying Cheng and Gavin Conibeer, Thin Solid Films, 520 (2011) 837.

[40] Brus L.E., J. Chem. Phys., 80 (1984) 4403. 


\section{Tables}

Table 1: A comparison of state in which a very thin and thick SnS films exists in.

\begin{tabular}{ccc}
\hline & Thin film & Thicker film \\
\hline Grain Size & Large & Small \\
$\mathrm{E}_{\mathrm{g}}$ & Lower & Larger \\
\hline
\end{tabular}




\section{Figures Captions}

1. A representative Raman spectra of a $900 \mathrm{~nm}$ thick $\mathrm{SnS}$ film before and after annealing at termperatures indicated.

2. X-Ray Diffraction patterns of SnS films of thicknesses (a) 270, (b) 480, (c) 600, (d) 630 and (e) $900 \mathrm{~nm}$ after annealing at $373 \mathrm{~K}$.

3. Graphs show the variation of (A) lattice constants with film thickness (annealed) films. Diameter of representing points are of the order of error in the determination of lattice parameters. (B) Grain size with annealing temperature. Bars indicate the $\pm 1.5 \mathrm{~nm}$ error in determination of grain size. These results were obtained from the X-Ray Diffraction Patterns.

4. The variation in Raman $A_{g}$ and $B_{2 g}$ peak positions of annealed (373 K and $473 \mathrm{~K}$ ) SnS films with film thickness.

5. The $B_{2 \mathrm{~g}}$ peak position of Raman spectra varies with grain size. The graph shows shift of Raman peak to lower energy level with increasing grain size.

6. SEM micrographs show grain size improvement in thinner film $(480 \mathrm{~nm})$ when annealed at higher temperature. However, the $900 \mathrm{~nm}$ films show no variation in grain size on annealing.

7. Energy Dispersive X-Ray (EDX) Spectroscopy of a larger area of $900 \mathrm{~nm}$ thick film, vacuum annealed at $473 \mathrm{~K}$, show no oxidation of the sample. The minimal Silicon and Oxygen present are from the substrate.

8. The band-gap of annealed SnS films were found to decrease with increasing grain size. The curve fit line agrees with theoretical model that explains variation of band-gap with grain size. 


\section{Figures}

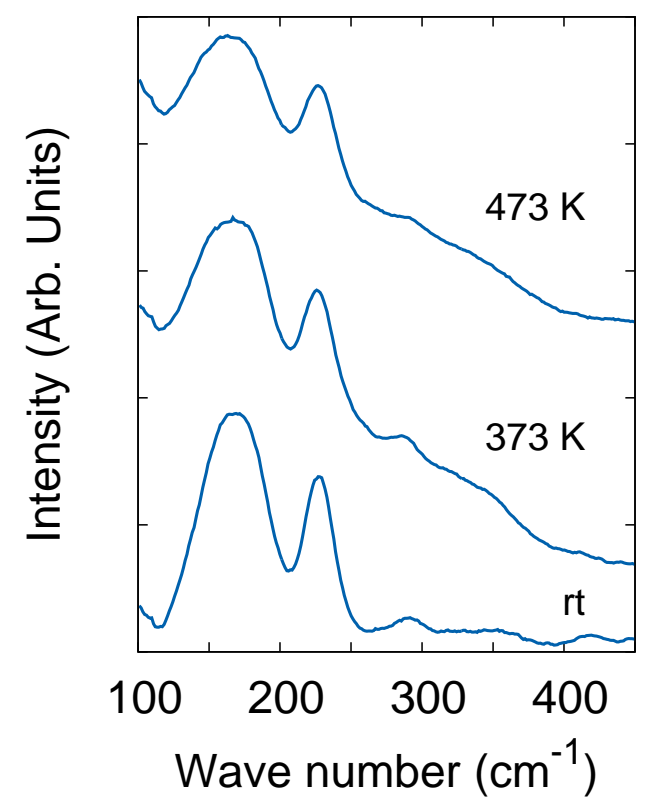

Figure 1: A representative Raman spectra of a $900 \mathrm{~nm}$ thick $\mathrm{SnS}$ film before and after annealing at termperatures indicated. 


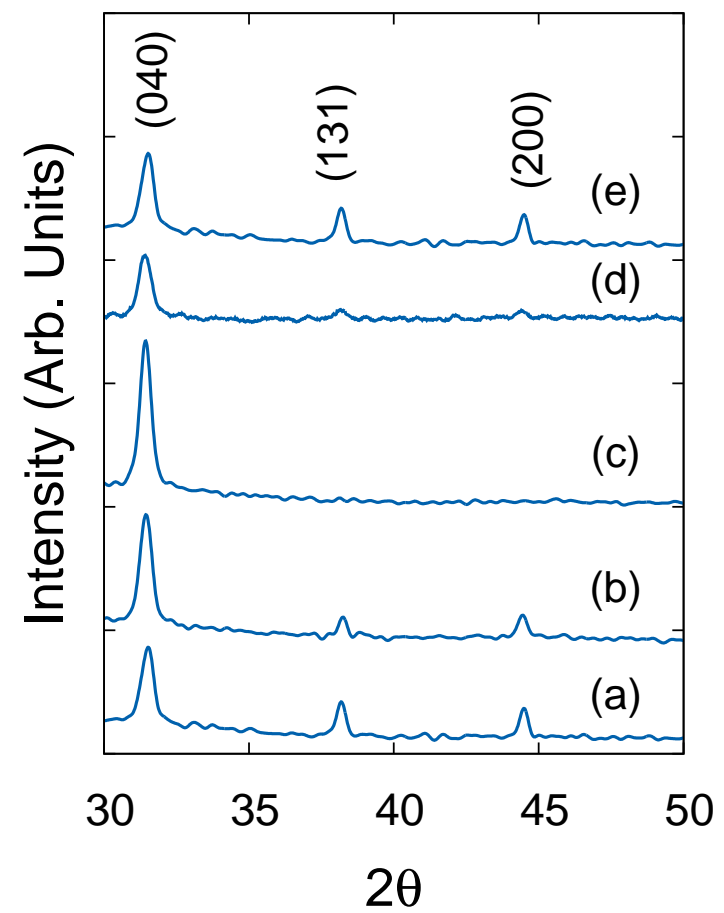

Figure 2: X-Ray Diffraction patterns of $\mathrm{SnS}$ films of thicknesses (a) 270, (b) 480, (c) 600, (d) 630 and (e) $900 \mathrm{~nm}$ after annealing at $373 \mathrm{~K}$. 

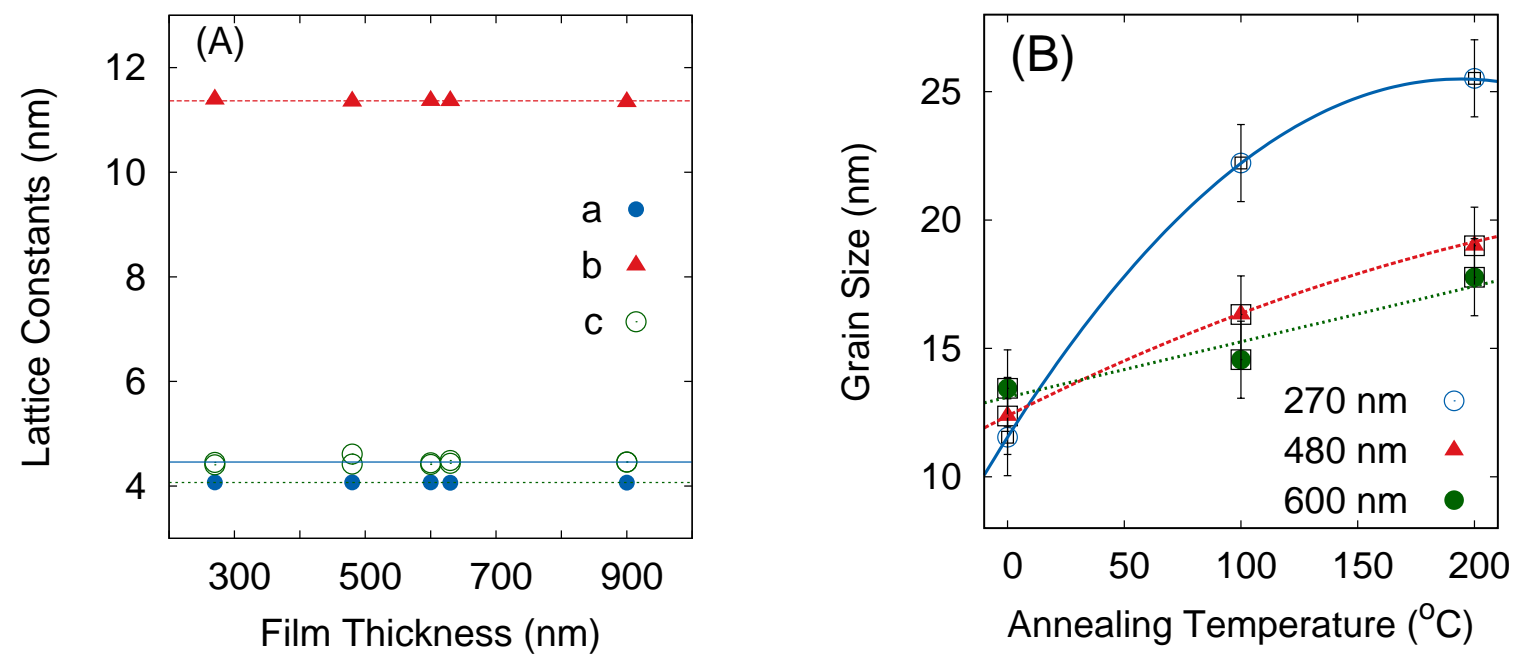

Figure 3: Graphs show the variation of (A) lattice constants with film thickness (annealed) films. Diameter of representing points are of the order of error in the determination of lattice parameters. (B) Grain size with annealing temperature. Bars indicate the $\pm 1.5 \mathrm{~nm}$ error in determination of grain size. These results were obtained from the X-Ray Diffraction Patterns. 


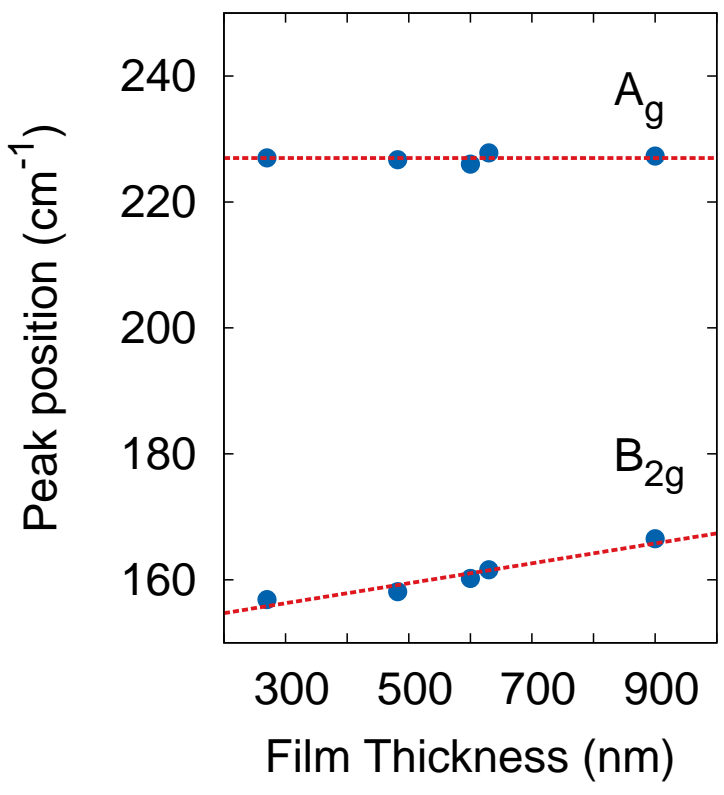

Figure 4: The variation in Raman $\mathrm{A}_{\mathrm{g}}$ and $\mathrm{B}_{2 \mathrm{~g}}$ peak positions of annealed ( $373 \mathrm{~K}$ and $473 \mathrm{~K}$ ) SnS films with film thickness. 


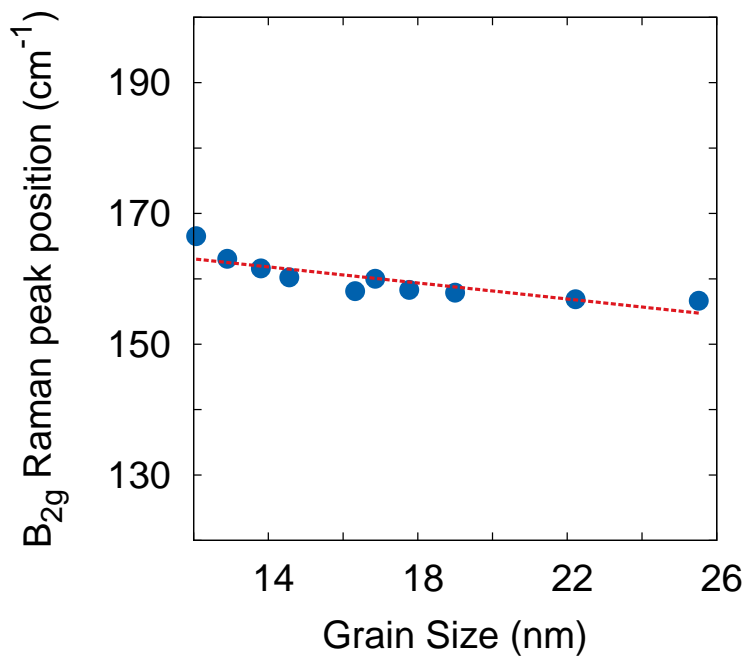

Figure 5: The $\mathrm{B}_{2 \mathrm{~g}}$ peak position of Raman spectra varies with grain size. The graph shows shift of Raman peak to lower energy level with increasing grain size. 

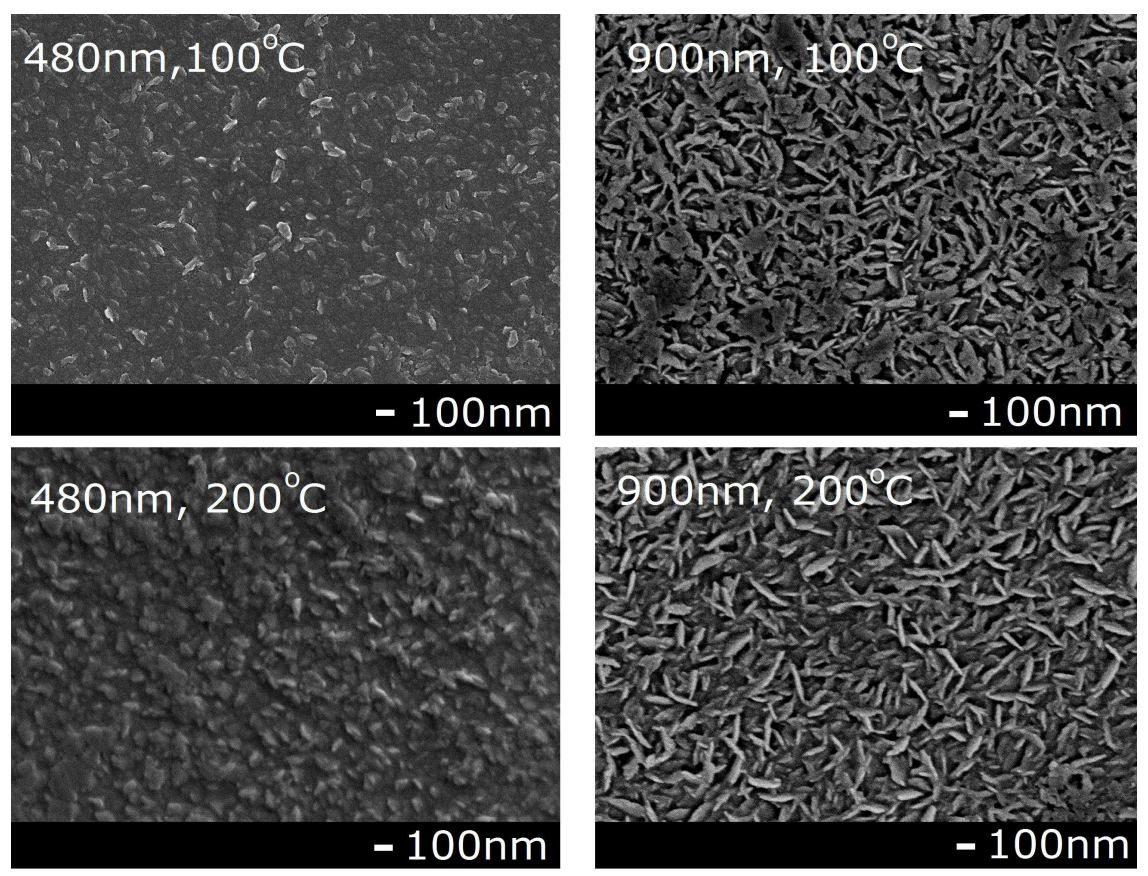

Figure 6: SEM micrographs show grain size improvement in thinner film $(480 \mathrm{~nm})$ when annealed at higher temperature. However, the $900 \mathrm{~nm}$ films show no variation in grain size on annealing. 


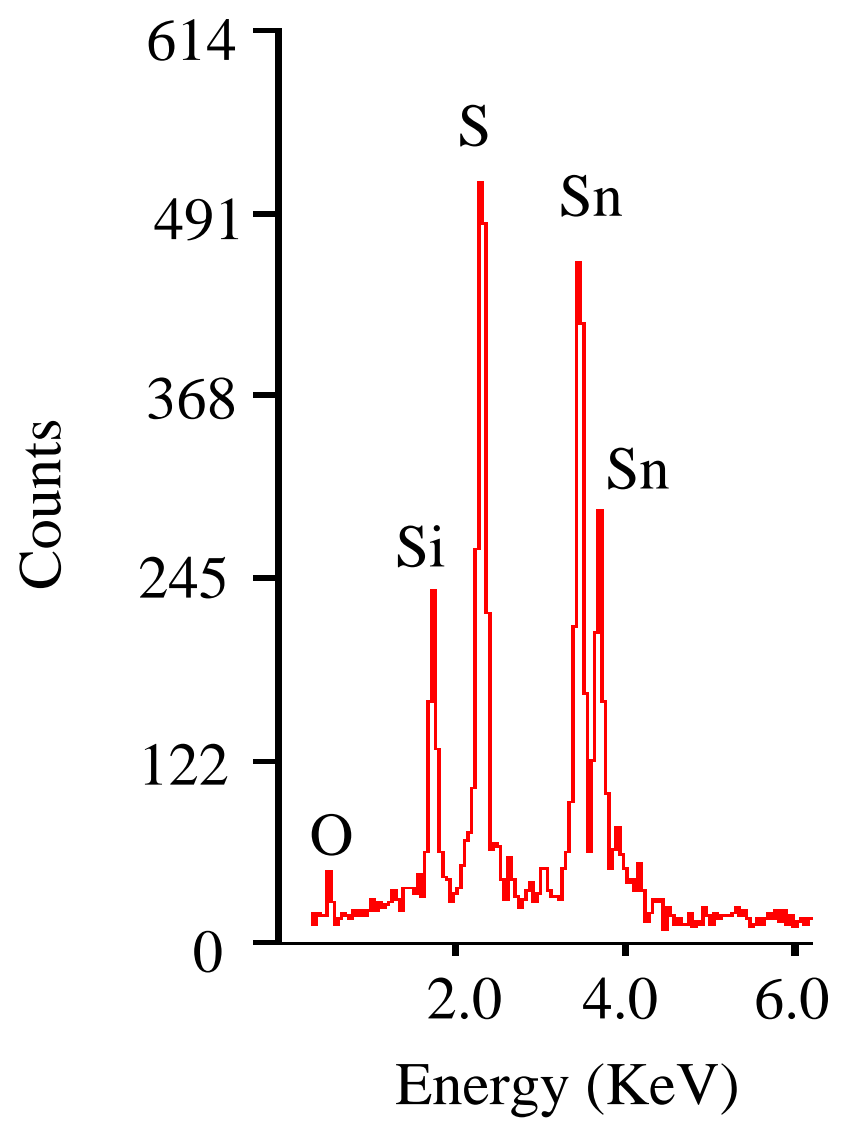

Figure 7: Energy Dispersive X-Ray (EDX) Spectroscopy of a larger area of $900 \mathrm{~nm}$ thick film, vacuum annealed at $473 \mathrm{~K}$, show no oxidation of the sample. The minimal Silicon and Oxygen present are from the substrate. 


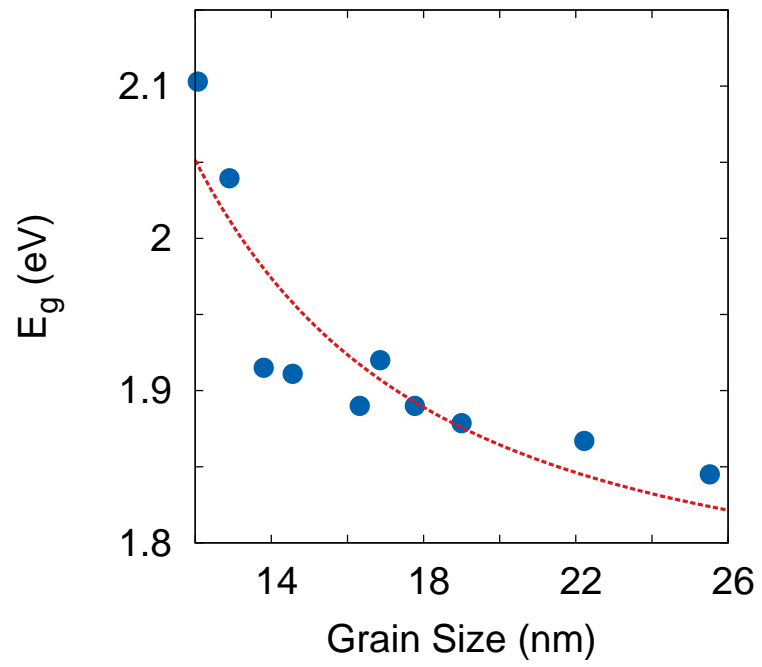

Figure 8: The band-gap of annealed SnS films were found to decrease with increasing grain size. The curve fit line agrees with theoretical model that explains variation of band-gap with grain size. 